Service social

\title{
Quelles sont les alliances possibles entre les décideurs, les acteurs et les usagers de l'action sociale? Ou comment se construit une politique sociale locale
}

\section{Catherine Manson}

Volume 46, numéro 2-3, 1997

Groupes - Symposium 1997

URI : https://id.erudit.org/iderudit/706764ar

DOI : https://doi.org/10.7202/706764ar

Aller au sommaire du numéro

Éditeur(s)

École de service social de l'Université Laval

ISSN

1708-1734 (numérique)

Découvrir la revue

Citer cet article

Manson, C. (1997). Quelles sont les alliances possibles entre les décideurs, les

acteurs et les usagers de l'action sociale? Ou comment se construit une

politique sociale locale. Service social, 46(2-3), 147-165.

https://doi.org/10.7202/706764ar 


\section{Quelles sont les alliances possibles entre les décideurs, les acteurs et les usagers de l'action sociale? \\ Ou comment se construit une politique sociale locale}

Catherine MANSON

\section{INTRODUCTION}

En tant que travailleurs sociaux, nous participons à la mise en œuvre d'une politique qui a été décidée; mais nos pratiques professionnelles nous permettent aussi de faire ressortir des besoins, pour faire évoluer cette politique. Je vous présente ici une recherche que j'ai effectuée dans le cadre d'une maîtrise à l'université de Paris-Nanterre, qui analyse le processus de décision d'élus locaux d'un département en France. À partir des résultats, nous réfléchirons à la place des élus, des services, des professionnels, mais aussi des usagers, dans le processus de décision. Après avoir d'abord situé le contexte institutionnel, nous verrons l'importance qu'il prend dans la prise de décision. Puis, à travers l'analyse de décisions successives dans un domaine de l'action sociale, nous mettrons en évidence les éléments utilisés par les élus pour déterminer une politique. Nous verrons enfin une pratique de travail social de groupe, comme moyen de replacer les usagers dans le processus décisionnel. 


\section{LE CONTEXTE INSTITUTIONNEL ET SON IMPORTANCE}

Dans le domaine de la politique sociale, des usagers, citoyens et électeurs, formulent des demandes et expriment des besoins; des élus prennent des décisions; des acteurs, services publics et opérateurs associatifs, mettent en œuvre les décisions. C'est le schéma simple et logique que l'on peut faire de la construction d'une politique sociale. Et ce schéma fonctionne dans des institutions. En France, il existe quatre niveaux institutionnels politiques, qui ont chacun un organe législatif (une ou plusieurs assemblées d'élus) et un organe exécutif :

- l'État, au niveau national, comprenant deux assemblées d'élus qui votent les lois et un Conseil des ministres chargé de l'exécutif, dirigé par un premier ministre et présidé par la Président de la République;

- la région (22 régions en France), avec une assemblée nationale d'élus, le Conseil régional, et le président de ce conseil, responsable de l'exécutif;

- le département (95 départements plus 5 départements d’outremer), avec un Conseil général, assemblée départementale d'élus, et le président de ce conseil, chargé de l'exécutif;

- la commune (38 000 communes environ), avec un conseil municipal d'élus, organe législatif, présidé par le maire et responsable de l'exécutif.

Chacun de ces quatre niveaux a des domaines de compétence spécifiques. Et ce qui a marqué en France, durant ces quinze dernières années, les services sociaux (mais pas seulement eux) est ce que nous appelons la décentralisation. C'est un ensemble de lois qui ont profondément modifié le niveau de décision dans plusieurs domaines de compétences, et en particulier celui de la politique sociale. Avant ces lois mises en œuvre à partir des années 19831984, c'est l'État qui non seulement déterminait la politique sociale nationale, mais aussi prenait les décisions concernant la politique sociale locale. Ainsi, toute création, extension ou suppression de services sociaux relevait du ministère des Affaires sociales. Ce qui, compte tenu de la distance à la fois géographique et en temps, ne favorisait pas l'adéquation entre les besoins sur le terrain et les 
moyens accordés. À cette époque aussi, la plupart des services sociaux publics dépendaient de l'administration de l'État.

En matière sociale, la décentralisation a permis le transfert des pouvoirs de l'État vers les départements caractérisés par :

- des transferts de compétences donnant des moyens juridiques à l'échelon local;

- des transferts financiers de l'État vers les collectivités locales, entraînant une responsabilité financière des élus locaux le " qui décide, paie » de M. Girard, directeur de l'Action sociale en 1983);

- des transferts de services et de personnel; c'est ainsi que de nombreux travailleurs sociaux ont changé d'employeur.

Quels étaient les objectifs de la décentralisation?

- rapprocher la décision du terrain : l'échelon départemental semblait le mieux placé pour appréhender les besoins sociaux et y répondre efficacement; par ailleurs, les services sociaux de l'État déjà bien structurés à l'échelon départemental pouvaient facilement être transférés;

- libérer le pouvoir des élus locaux de la tutelle de l'État, objectif démocratique de la réforme;

- rapprocher les pouvoirs publics des citoyens.

Le secteur social n'a pas été le seul domaine transféré au cours de la décentralisation, mais ce fut le plus important en termes financiers et en quantité de personnel. Cette réforme a eu lieu - et certains disent que ce ne fut pas un hasard - dans un contexte économique difficile: les crises économiques successives ont accentué les problèmes sociaux, donc augmenté les dépenses dans ce domaine, mais aussi réduit les capacités financières publiques. Les élus locaux ont alors l'obligation d'établir une politique sociale plus efficace avec un budget stable. Ce contexte institutionnel et économique caractérisé par une pression politique et financière sur les élus locaux entraînera tout au long des années 1980 et jusqu'au début des années 1990 une méfiance des élus départementaux visà-vis de ce qui est difficilement contrôlable et un refus de prendre en charge ce qui peut être assumé par d'autres; méfiance vis-à-vis des 
commissions d'attribution de certaines prestations, vis-à-vis de certaines associations en remettant en cause les conventions, report de charge sur l'État ou d'autres collectivités locales lorsque l'action touche plusieurs domaines de compétence (la protection de l'enfance et la justice, par exemple) ou une catégorie de population (les nomades). II est, par ailleurs, des secteurs plus ou moins propices aux restrictions budgétaires, comme les interventions dont les objectifs sont peu explicités et les résultats difficilement mesurables (telles certaines actions de prévention), des secteurs où la mobilisation sociale est faible et l'impact politique négligeable. Par contre, des domaines seront privilégiés parce qu'ils touchent des bénéficiaires influents politiquement (personnes âgées, personnes handicapées...). Certaines actions, comme la création d'un équipement, peuvent avoir un impact politique, parce qu'elles rendent le pouvoir plus visible et renforcent une image positive du département.

Ce contexte ne sera donc pas sans conséquences pour ceux qui mettent en œuvre les décisions, à savoir les services et les associations. Faisant référence aux travaux de Michel Crozier (1963), Rodier (1987) met en évidence la position stratégique des principaux acteurs de l'action sociale, à partir de leurs fonctions d'expertise, de contrôle des règles, de contrôle de l'information ou de contrôle des moyens financiers. C'est ainsi que la source du pouvoir des élus dans le domaine de l'action sociale se situerait au niveau des moyens financiers et d'une certaine maîtrise de l'information, nécessaire pour décider « juste ». Les cadres administratifs auraient la maîtrise des règles : ils instruisent les demandes et contrôlent la mise en œuvre des décisions; leur capacité à interpréter la règle leur permet d'agir sur la décision. lls se considèrent comme des " techniciens » auprès des élus. Les professionnels, travailleurs sociaux des services départementaux et des associations, mettent en œuvre les décisions des élus. Ils ont la maîtrise de l'information issue du terrain, des données sur les besoins et des effets des actions. Le changement de décideurs a modifié les stratégies des uns et des autres. D'une logique administrative, les décisions sont passées à une logique politique et les acteurs ont dû alors recomposer leurs jeux. Si les élus se méfient des professionnels et des associations, ils s'assurent le concours fidèle des services, pourvoyeurs d'informations. « Le pouvoir public définit une politique et monnaye celle-ci en actions qu'il est prêt à financer » (Gontcharoff, 1988). 
Pour mettre en œuvre leurs décisions, les élus ont le choix entre le " faire " ou le " faire-faire "; ils ont le choix des opérateurs, c'est-à-dire le service direct (par le personnel départemental) ou les opérateurs du secteur associatif. Or, au moment de la décentralisation, l'action sociale était mise en œuvre à $80 \%$ par des associations. Les projets émanaient le plus souvent des associations qui les montaient et cherchaient à les "vendre " en faisant appel à l'État, principal interlocuteur et pourvoyeur de fonds. Depuis la décentralisation, le mécanisme de négociation est inversé. Ce sont les élus qui sont demandeurs vis-à-vis des associations : dans une logique d'appels d'offres faits aux opérateurs, ils fixent les implantations géographiques, déterminent des priorités, demandent des comptes. Ils veulent par ailleurs affirmer leur pouvoir, faire une reprise en main. C'est ainsi que les associations sont vite amenées à apporter la preuve de leur légitimité technique et professionnelle et à prouver l'efficacité de leurs actions. Avant la décentralisation, la logique administrative aboutissait plus à une logique de moyens que de résultats : le fonctionnaire, représentant de l'État, n'avait pas de comptes à rendre à des contribuables (anonymat de l'argent public), le suivi des conventions était plutôt formel. Depuis la décentralisation, il y a passage d'une logique administrative à une logique politique et à une logique de résultats : “ L'élu politique a des comptes à rendre aux contribuables, donc des comptes à demander aux opérateurs. L'enjeu pour lui est de faire la démonstration publique de l'excellence de ses choix » (G. Gontcharoff, 1985).

La logique associative est de défendre son projet et d'en trouver le financement; les associations ont ainsi toujours plus ou moins été subordonnées aux financeurs. C'est pourquoi, progressivement, elles ont adopté une structure pyramidale pour mieux négocier avec celle des pouvoirs publics centralisés; elles se sont spécialisées en fonction des services administratifs et des prestations; elles se sont habituées à la reconduction quasi systématique des subventions. Mais la décentralisation a rendu inadéquate leur structure pyramidale et les niveaux départementaux ont dû apprendre à négocier. Parfois leurs missions se sont retrouvées sur deux domaines de compétence, relevant de deux interlocuteurs différents qui se renvoient le financement l'un à l'autre. Je pense en particulier à une association qui a pour mission l'accompagnement des familles de détenus avec pour objectif d'éviter l'éclatement du système familial, 
du fait de l'éloignement d'un parent, et d'éviter surtout le placement des enfants. Cette association a mis plus de deux ans pour convaincre les élus départementaux que sa mission relevait, certes, de l'État de par sa mission liée à la détention donc du domaine de la justice, mais aussi du Conseil général, à travers la protection de l'enfance.

Ainsi, les associations - opérateurs à $80 \%$ dans l'action sociale - ont été bousculées par les changements de décideurs : elles ont subi de plein fouet les prérogatives des nouveaux décideurs départementaux, le plus souvent sans négociation possible; car pour survivre et maintenir leurs emplois elles n'ont pas eu d'autre possibilité que d'accepter: "Le pouvoir politique sait que si l'association ne signe pas elle disparaît » (Gontcharoff, 1988).

Le changement d'interlocuteurs bouscule donc les mentalités, les anciennes façons de faire entre les décideurs et les acteurs. Si la décentralisation a permis aux associations de clarifier leurs rapports avec les collectivités locales, elle a révélé leurs faiblesses devant le pouvoir décisionnel. Pourtant, un des grands principes annoncés est de rapprocher les élus des citoyens, d'élaborer des programmes sociaux adaptés aux besoins locaux. Mais les élus ont besoin de données pour effectuer des choix et pour les justifier. Quels rapports vont-ils entretenir avec les détenteurs de l'information et quelles formes de savoir vont-ils privilégier pour prendre une décision?

Cette présentation du contexte institutionnel est tout à fait nécessaire pour comprendre le processus décisionnel des élus, de même que la façon dont les professionnels du travail social se situent dans ce contexte et par rapport aux décideurs. Pour savoir, aussi, comment faire évoluer la place des usagers-citoyens dans ce processus.

\section{LA PRISE DE DÉCISION POLITIQUE}

II s'agit de mettre en évidence comment les élus décident et à partir de quels savoirs. Comment se placent les savoirs issus du terrain, produits par des professionnels? Qu'est-ce qui motive leurs choix? Comment sont perçues les demandes et les attentes des usagers? Quel est le sens des décisions prises dans un domaine social?

Pour cela, nous verrons brièvement le circuit décisionnel des élus dans lequel nous retrouvons l'assemblée des élus départementaux ou Conseil général, présidé par un des leurs, élu par l'assemblée. 
Théoriquement, l'assemblée a le pouvoir législatif; c'est elle qui décide. Or, le président a un rôle très important : il fixe l'ordre du jour des débats à l'assemblée; il présente un rapport pour chaque point de l'ordre du jour et formule la décision sur laquelle les élus doivent débattre et se prononcer en l'adoptant, la rejetant ou la renvoyant pour un examen complémentaire. Nous voyons que les élus au conseil ne peuvent débattre que dans le cadre étroitement fixé par leur président. Celui-ci, pour assumer son rôle, est assisté d'un pôle technique: des services administratifs dirigés par un directeur général nommé par le président de l'assemblée. Ce pôle technique informe le président des problèmes sociaux, propose des solutions et les argumente. Une décision votée engendre le plus souvent une dépense publique et, une fois les crédits débloqués, c'est le pôle technique qui est chargé de la mettre en œuvre, soit par ses propres services, soit en faisant appel à un opérateur externe. Ainsi, les services jouent un grand rôle dans ce processus décisionnel, puisqu'ils sont à la fois à l'amont et à l'aval de la prise de décision. Les élus peuvent, cependant, avoir leur propre source d'information, par leur participation, à titre personnel ou du fait même de leur mandat, aux conseils d'administration d'associations, d'hôpitaux, d'établissements scolaires. De plus, la moitié des conseillers généraux sont aussi maires, donc plus proches des problèmes locaux (ce qui risque de changer prochainement du fait d'un projet de loi visant à limiter le cumul des mandats). Malgré cela, un fossé semble se creuser entre les élus départementaux et les citoyens. En effet, ces derniers participent de moins en moins à l'élection des conseillers généraux; ils ignorent le rôle de ces élus, surtout en milieu urbain où le conseiller a peu de contacts directs avec les usagers.

Le champ de la politique sociale du département, objet des décisions, est très vaste. J'ai donc limité ma recherche à un secteur particulier, celui de la prévention spécialisée. Pourquoi ce choix?

- Sur le plan méthodologique, il existait plusieurs rapports successifs traitant exclusivement de cet objet sur la période considérée (1983, date de début de la décentralisation dans le domaine social, jusqu'en 1993).

- Sur le plan des hypothèses de travail, la prévention spécialisée fut l'un des premiers objets de décision des élus, dans le domaine de l'action sociale; 
la prévention spécialisée met en œuvre des interventions éducatives auprès des jeunes sur des secteurs géographiques en voie de devenir "sensibles "; les actions prennent des formes diverses en fonction de la problématique des jeunes, de leur milieu de vie, des finalités des associations et des compétences particulières des professionnels;

la mise en œuvre sur le terrain de ce domaine est effectuée par des associations;

les effets des actions sont difficilement mesurables et s'évaluent à long terme, ce qui peut en faire un terrain d'enjeux politique et idéologique;

la prévention spécialisée faisait partie à l'époque du chapitre budgétaire de l'Aide sociale à l'enfance, jugé trop lourd et objet d'enjeux financiers.

Dans le cadre de la recherche que j'ai effectuée, j'ai analysé le contenu des rapports, et les débats sur ces rapports. Les rapports étudiés sont des documents écrits; les débats sont des interventions verbales des élus réunis en assemblée, mais retranscrites in extenso. Si les auteurs des discours exprimés au cours des débats se confondent avec leurs énonciateurs, l'auteur des rapports n'en est pas forcément le signataire. En effet, nous avons vu plus haut que le président donne son avis et signe les rapports, mais il n'en est pas forcément l'auteur; ces rapports sont le plus souvent élaborés par les services. C'est par l'analyse de discours, discipline connexe aux sciences du langage, que j'ai choisi d'étudier cet ensemble de textes. Mon objectif était de déterminer le contenu des documents et les intentions des locuteurs, c'est-à-dire la signification du discours. Dans cette méthodologie de recherche, le cadre de l'énonciation, les sujets énonciateurs, l'objet sur lequel porte le discours importent. Nous verrons que l'analyse de discours est plus un travail d'explicitation que d'explication, qui laisse entrevoir un autre discours à l'œuvre à travers le premier. Tout fragment de discours suppose que le locuteur a opéré un choix qui est significatif. L'élaboration des politiques publiques, exercice du pouvoir des élus, consiste en « un flux de décisions et de procédures, dont il faut essayer de trouver le sens [...] les élus décident en fonction de leur champ cognitif et de la structuration du système de décision, c'est-à-dire du 
mode de formulation du problème et des différentes options ". Le champ cognitif de l'élu (ou les informations qu'il juge pertinentes) constitue un filtre pour simplifier et structurer le champ de la décision. Il est limité par sa propre représentation du problème et par la structure de décision, réduite à ce que lui laissent les médiateurs, ceux qui cernent le problème, le formulent et en préparent la proposition de solution.

Ayant étudié le processus de décision dans un département, nous avons mis en évidence l'importance des services dans le circuit de décision : les rapports rédigés par les services sont une identification et une formulation du problème, décision qui doit être prise, qui s'impose aux élus. "Élaborer une politique publique revient à construire une représentation, une image de la réalité sur laquelle on veut intervenir. C'est en référence à cette image cognitive que les acteurs vont organiser leur perception du système, confronter leurs solutions et définir leurs propositions d'action: on appellera cet ensemble d'images le référentiel d'une politique " (Muller, 1994). Ce référentiel, toujours d'après Pierre Muller, se construit en fonction :

- de l'image que s'en font les acteurs,

- des normes et valeurs de référence,

- de la place et du rôle du secteur concerné par la décision dans la société globale,

- du médiateur qui opère la construction et la transformation du référentiel.

Le médiateur a une position stratégique dans le système de décision et j'ai posé comme hypothèse qu'il se situait au niveau des services administratifs. Pour vérifier cette hypothèse, les textes - rapports et débats - ont été traités d'abord par une analyse de contenu, lecture linéaire, pour déterminer la place de l'argumentation technique par rapport à l'argumentation idéologique ou politique. Puis j'ai effectué une analyse de l'énonciation pour mettre en évidence les marques implicites du discours qui donnent un sens à l'objet et sont des marques d'enjeux de pouvoir entre les acteurs (décideurs, services, professionnels). En effet, ce n'est pas forcément ce qui est dit explicitement qui donne le sens, mais la façon dont cela est dit, la façon dont le discours est construit. Enfin, j’ai traité les rapports par 
une analyse lexicométrique, c'est-à-dire une étude du vocabulaire utilisé afin de déterminer et de compléter le sens des décisions.

Disons d'ores et déjà que l'argumentation développée dans les rapports est différente de celle menée par les élus au cours des débats. Les rapports présentent des décisions qui ont des orientations politiques, que l'accord de la section spécialisée (organe de concertation sur la protection de l'enfance entre des élus, des professionnels, des responsables de services et d'associations), complété par des études statistiques, suffit à justifier. Cependant, la décision politique et sa justification technique s'articulent mal, comme deux discours qui fonctionnent côte à côte; l'argumentation technique servant d'alibi, de faire-valoir à l'orientation politique, sans réellement la justifier. Par contre, l'argumentation développée par les élus qui interviennent dans les débats est précise et centrée sur les problèmes rencontrés sur le terrain; en général, elle s'articule avec l'objet du débat. Prenons un exemple : des besoins nouveaux apparaissent dans un secteur du département qui exigerait de mettre en place une nouvelle équipe de prévention. Cependant, les orientations politiques générales imposent de ne pas augmenter les dépenses du département dans ce domaine : la proposition est de redéployer les équipes; si une équipe est nécessaire là, il faut en supprimer une ici; ou faire participer les communes au financement des actions afin d'accroître les possibilités sans augmenter les dépenses du département. Les élus au cours des débats relèvent le manque d'argumentation technique; ils demandent des discussions de fond sur la prévention et regrettent le manque de concertation avec les personnes sur le terrain, se centrent sur le contenu des actions sociales.

L'analyse du vocabulaire employé dans la formulation d'une succession de décisions et de leurs argumentations met en évidence :

- les formes d'action du Conseil général dans le domaine de la prévention spécialisée, soit le financement, la réorganisation; la restructuration, l'agrément et le déconventionnement;

- les moyens d'action, soit la subvention, le contrôle, le suivi; l'évaluation, les études, les bilans, la concertation, la négociation.

Cette analyse permet d'établir ce qu'on appelle une grille actancielle, qui détermine comment les différents acteurs concernés 
dans les décisions sont représentés dans le discours. Ainsi, celui qui agit, le sujet, est le Conseil général. L'objet de l'action concerne les équipes de prévention et leurs actions. Le destinataire, ce sont les jeunes (adolescents et jeunes adultes), issus de milieux défavorisés ou de milieux où les phénomènes d'inadaptation sociale sont particulièrement développés, les zones sensibles. L'adjuvant, celui qui aide, est la section spécialisée du conseil départemental de protection de l'enfance; celle-ci est de plus en plus présente dans les discours des élus, qui la consultent sur les difficultés des équipes, sur les besoins, sur l'évaluation des moyens et des réalisations. L'opposant semble être les associations, suspectées, et qui sont l'objet de contrôle. Cette grille actancielle met en évidence les éléments utilisés par les élus pour agir : elle montre une volonté de maîtriser les actions des équipes de prévention, le lieu de leurs actions et leurs acteurs. Elle se caractérise par un contrôle des associations, gestionnaires des fonds, perçues comme des rivales. Les élus, pour justifier leurs décisions et contrebalancer le pouvoir des associations, s'appuient sur la compétence technique de la section spécialisée, organe de concertation, et sur les principes de la prévention spécialisée, principes élaborés par les professions de l'éducation spécialisée et reconnus par elles.

Les données fournies par la lecture linéaire des rapports et des débats ainsi que par l'analyse du vocabulaire mettent en évidence que le référentiel des décisions est essentiellement d'ordre technique et que les médiateurs (ceux qui opèrent la construction et la transformation du référentiel) sont principalement les services. Les énoncés d'ordre politique donnent le sens des décisions et renvoient une certaine image de l'objet de décision; ainsi, dans les rapports, la prévention spécialisée est présentée comme un secteur de l'action sociale dispendieux, dont il faut maîtriser les dépenses. Mais c'est aussi un secteur dont il faut contrôler le fonctionnement et les activités. L'argumentation technique semble venir soutenir les décisions : la présence des textes juridiques et des principes de la prévention spécialisée, dans les rapports, est une façon de dédouaner les décideurs; les statistiques rationalisent les décisions; parfois, la description des activités développées sur le terrain justifie leur adéquation avec les problèmes locaux. Cette dernière forme d'argumentation est utilisée pour donner en exemple certaines associations (d'autres, dans le même temps, sont déconventionnées pour " mauvaises 
conduites "). Les élus souhaitent des associations qui sollicitent un financement complémentaire des communes, qui participent aux réunions de concertation de la section spécialisée, qui incluent dans leur conseil d'administration des élus, qui ont des contacts réguliers avec eux, qui adhèrent à la politique départementale. Le médiateur semble bien être les services : ils informent et forment les élus, surtout au début de la décentralisation, sur leurs nouveaux domaines de compétence. Placés entre les décideurs et les acteurs de terrain, ils fournissent les informations aux élus. Cependant, les arguments fournis sont mis en forme, sélectionnés, en fonction des orientations politiques générales.

La lecture des débats montre que les élus qui interviennent ont un discours centré sur des éléments concrets de leur territoire. Ces faits sont quelquefois en contradiction avec les arguments avancés dans les rapports. Les élus, en tant que conseiller général, maire, membre de la section spécialisée ou d'un conseil d'administration d'une association, apportent des informations du terrain, tirées de leur expérience dans le domaine touché par la décision. Leur discours paraît alors loin de celui du rapport, mais plus en phase avec l'objet de décision, mettant parfois le président en contradiction. Malgré cela, les élus de l'assemblée semblent peu faire évoluer le référentiel des décisions ou seulement lorsque les orientations politiques générales le permettent.

L'analyse de la forme du discours apporte d'autres éléments sur le processus de décision, notamment sur les rapports de pouvoir au sein de la structure institutionnelle et combien ce contexte ritualise et fige les rapports entre les élus, plus particulièrement entre le président et les autres membres de l'assemblée. Le président manifeste son pouvoir à travers les rapports : décisions et argumentations sont présentées de telle manière que ces rapports paraissent indiscutablement vrais et irréfutables; l'avis de la section spécialisée est utilisé comme une garantie de validité, comme un « label ». Les élus de l'assemblée manifestent leur pouvoir légitime en évoquant leur statut, de conseiller général, maire, membre de conseil d'administration; ils s'expriment en s'impliquant personnellement, rompant avec le discours dépersonnalisé des rapports. " Je peux prétendre bien connaître le sujet », dit l'un d'entre eux, par exemple. Je poserai comme hypothèse que cette forte implication est une façon pour les élus de reprendre leur fonction décisionnelle 
face au président qui ne leur laisse aucune marge de manœuvre. Les élus chercheraient à se réapproprier le pouvoir de décider, non sur le plan institutionnel, mais sur le plan de leurs compétences.

Les résultats ont aussi montré une volonté de pouvoir des élus sur les acteurs associatifs. Les associations ont pourtant pour fonction, dans le champ des politiques sociales, de maintenir un contact entre les usagers, les professionnels et les décideurs. Proches du terrain et des usagers, elles sont une force de propositions pour des actions nouvelles et adaptées aux besoins sociaux en constante évolution. Elles ne veulent pas être seulement considérées comme prestataires de services. Or, l'analyse des décisions successives montre que les élus cherchent à se réapproprier cette force de proposition, considérée comme une zone de pouvoir au niveau des usagers. Un jeu complexe s'établit entre les associations et les pouvoirs publics, d'autant plus complexe que les associations n'acquièrent de pouvoir que si elles sont reconnues par les pouvoirs locaux. Les rapports des élus avec les associations sont souvent conflictuels : contrairement aux rapports des élus avec les techniciens qui peuvent trouver un compromis en reconnaissant leur propre légitimité, les élus et les associations se battent sur le même terrain. Ce sont deux forces de propositions pour répondre aux problèmes sociaux; elles se situent toutes deux dans le champ politique, donc dans un rapport de force. Elles utilisent les mêmes ressources : une assise sociale, une base militante, un réseau de notables, un savoir-faire.

Cette recherche montre une volonté de la part des élus de maîtriser la mise en œuvre des actions sociales, en diminuant la marge de manœuvre des associations; les services, transformant les informations produites par les acteurs du terrain, feraient écran à la confrontation des professionnels avec les décideurs. Plusieurs études sur les effets de la décentralisation vont dans le même sens : J.M. Ohnet (1996) explique que la décentralisation a ouvert un champ politique local spécifique, mais « qu'il est le produit d'un modèle centralisé et l'expression d'une culture unitaire ". II ajoute que la décentralisation confirme la reproduction du modèle centralisé aux échelons locaux, caractérisés par " une interpénétration entre les domaines administratif et politique qui se manifeste par un contrôle croisé des pouvoirs administratif et politique, au détriment de l'indispensable médiation du politique avec les réseaux locaux, notamment les citoyens et les associations. [...] Les élus ont manifesté 
dès le départ une certaine méfiance à l'égard des discours, parfois idéologiques, tenus sur l'action sociale. Attendant des résultats concrets et préférant des actions, même limitées ou ponctuelles, susceptibles d'entraîner des effets immédiatement mesurables et politiquement exploitables, les élus ont modifié le mode d'emploi du service social. »

Depuis quelques années déjà se dessinent cependant des initiatives pour ne pas perdre cet atout majeur que représente le contact avec les usagers. Au niveau national comme au niveau local, dans certains départements, des commissions se mettent en place pour définir les rôles des élus et ceux des associations, dans le domaine de l'action sociale, dans le sens d'un renforcement du processus démocratique, c'est-à-dire en considérant les associations comme des partenaires et non comme de simples opérateurs. Des observatoires de l'action sociale sont mis en place à la demande des élus pour les aider à décider en concertation avec les partenaires de l'action sociale. Cela témoigne, espérons-le, d'une volonté de dépasser les enjeux de pouvoir pour décider au plus près des besoins.

\section{UNE PRATIQUE DE TRAVAIL SOCIAL AVEC UN GROUPE}

\section{Comme moyen de resituer le discours des usagers dans la décision politique}

Portant un grand intérêt au travail social avec les groupes, j'ai remarqué que certaines pratiques de travail social avec des groupes d'usagers permettaient de modifier ou d'affiner la représentation que pouvaient avoir les élus des problèmes sociaux et ainsi participer à la construction du référentiel des décisions.

Ainsi, j'ai choisi de terminer mon intervention par la présentation de l'une de ces pratiques, qui m'est apparue particulièrement significative, car elle se situe dans le contexte institutionnel que je viens de décrire : elle montre comment un service peut évoluer tout en gardant sa spécificité et comment, à la faveur d'un changement, il peut mettre en place des pratiques qui favorisent la prise en compte des besoins par les décideurs.

Il s'agit d'un service social de la Caisse d'allocations familiales (CAF) dans un département de province, qui était conventionné avec 
le département pour une mission de polyvalence de secteur. Dans le contexte de la décentralisation, les conseils généraux ont décidé, pour la plupart, d'assurer cette mission avec leurs propres services et de supprimer au fur et à mesure les conventions avec les services extérieurs. C'est ce qui est arrivé à ce service social de la CAF, alors que le personnel n'y était pas préparé.

Ce service a donc dû se réorganiser complètement pour maintenir son activité sur le département et garder son potentiel en personnel. Pour se définir de nouvelles missions et construire une organisation appropriée, il a élaboré des moyens logistiques en audit et en formation. D'un service de polyvalence il est devenu un service spécialisé avec des fonctions spécifiques en direction des familles et des jeunes, tout en gardant les finalités propres communes à l'ensemble des services sociaux de la Caisse nationale d'allocations familiales. Au cours d'un important travail de groupe des personnels administratifs et sociaux, au sein du service, et au vu d'une étude de besoins effectuée sur le département, les directions du travail social ont été déterminées :

- créer du lien social;

- favoriser l'innovation sociale;

- faciliter l'accès aux loisirs et aux vacances.

Les projets d'interventions sociales devaient prendre en compte les points suivants :

- développer les acquisitions et savoir-faire du personnel en direction des familles;

- contribuer à la notion de proximité en expérimentant de nouvelles implantations;

- développer un travail collectif par projet.

Les projets de travail collectif dont font partie, ici, les pratiques de travail avec les groupes devaient prendre certains critères en considération :

- $\quad$ s'inscrire dans les objectifs du service;

- $\quad$ répondre à des besoins repérés;

- $\quad$ respecter les usagers dans leurs valeurs et leur histoire; 
- développer la complémentarité des senvices dans un partenariat;

- tenir compte des compétences des personnels;

- évaluer le projet.

C'est ainsi que plusieurs projets ont émergé dans le service et ont été mis en œuvre sur le terrain. Ainsi, ce senvice de la CAF a créé un produit, au niveau du département, qui est le PasseportTemps Libre, sorte de chèque-temps libre destiné aux jeunes de 13 à 17 ans qui est utilisable auprès des associations conventionnées. Une étude effectuée par le service sur l'utilisation de ce produit a révélé qu'on ne l'employait pas dans certains secteurs du département. Le service a décidé d'en savoir plus et, sur deux sites, a proposé une réunion à l'ensemble des jeunes concernés. Ceux-ci ont été invités par courrier, puis relancés par téléphone. Les objectifs de la réunion étaient de permettre aux jeunes de donner leur avis sur le Passeport-Temps Libre, de s'informer éventuellement sur le produit, d'exprimer leurs besoins dans le domaine du temps libre.

Pour l'un des sites, une petite ville de 6000 habitants où le taux d'utilisation du Passeport-Temps Libre est très faible, une vingtaine de jeunes viennent à cette première réunion. Plusieurs problèmes y sont exprimés :

- une information inadaptée du produit;

- un décalage entre les activités proposées aux jeunes sur la ville et leurs besoins dans le domaine des loisirs;

- l'expression par les jeunes d'un ressentiment vis-à-vis des associations paramunicipales sur leur marque d'ouverture et de dialogue avec eux.

Des pistes de travail ont été décidées par les travailleurs sociaux du service CAF :

- travailler sur la plaquette d'information pour la rendre plus efficace; ce travail a été réalisé et repris par l'institution CAF;

- effectuer une enquête auprès des jeunes de la ville pour évaluer leurs besoins dans le domaine des loisirs.

L'intervention sociale a duré un peu plus d'un an : un travailleur social a travaillé avec ce groupe de vingt jeunes au sein duquel s'est 
dégagé un noyau moteur de sept jeunes; il a produit une aide technique pour que le groupe fonctionne et travaille à ses objectifs; du simple fait de sa présence, il a permis au groupe d'obtenir de la part de la mairie la mise à disposition d'une salle pour se réunir; il lui a permis l'utilisation des moyens techniques du service pour construire un questionnaire.

Au départ, les élus de la ville considéraient que les « revendications " des jeunes n'étaient pas justifiées, car selon eux il y avait déjà tout ce qu'il fallait pour répondre à leurs besoins. L'encadrement du projet d'action des jeunes par le service CAF a servi de caution auprès des élus et a permis que les jeunes continuent à se réunir. Le travailleur social a, tout au long de l'intervention, maintenu des contacts ponctuels mais réguliers, en accord avec le groupe, avec les élus pour clarifier son rôle et les informer du travail du groupe. Le questionnaire a pu ainsi être construit par le groupe et lancé auprès de 256 jeunes de la ville. On a obtenu 89 réponses, qui ont surtout mis en évidence le besoin d'un lieu ouvert d'accueil. Déjà, le collège avait fait ressortir ce besoin des jeunes, qui n'avait pas été entendu. Les jeunes ont alors décidé de rendre compte des résultats de l'enquête aux élus de la ville. Ceux-ci ont été surpris par la qualité du travail du groupe, sa capacité à se définir des objectifs et à s'organiser pour les atteindre, c'est-à-dire à prendre des responsabilités. Ce contact direct a modifié sensiblement la représentation que se faisaient les élus des jeunes de cet âge sur leur commune. Une écoute s'est manifestée ainsi qu'une volonté de reconnaître que des problèmes existaient dans le domaine des loisirs. Cela s'est concrétisé par la création d'un conseil municipal des jeunes, avec les associations, pour rétablir le contact et adapter les services proposés aux besoins exprimés. La municipalité n'a pas encore répondu à la demande initiale des jeunes de créer un lieu d'accueil qui leur permette de développer leurs propres activités. Cette action a aussi entraîné une réflexion dans l'institution CAF pour ouvrir l'utilisation du Passeport-Temps Libre : pourquoi ne pas l'utiliser comme soutien financier au démarrage d'un projet? Dans le cadre du projet des jeunes, cette possibilité offerte à la municipalité pourrait être une perche tendue pour qu'elle prenne position.

Cette action est intéressante à différents titres et son évaluation peut être effectuée sur plusieurs plans. Ce travail en groupe a permis à des jeunes de se resituer dans leur cité : partis de positions 
revendicatives isolées et passives, ils ont été amenés à prendre des rôles dans le groupe, à construire des outils d'action, à communiquer avec des élus et, surtout, ils ont redécouvert que " c'était possible ", même si leur demande n'a pas encore été totalement satisfaite. Les élus, de leur côté, décident dans le cadre institutionnel de la commune, avec leur propre représentation des problèmes et des usagers. Même si d'autres institutions (le collège, les services sociaux) les informent de l'existence de besoins, cela ne remplace pas et ne vaut pas une expression directe et travaillée d'un groupe d'usagers, premiers concernés par les décisions des élus.

D'autres pratiques pourraient illustrer encore le fait que, pour des personnes, contacter des élus, c'est se relier au système social, c'est redevenir un citoyen capable d'agir sur son système social, et nous, travailleurs sociaux, participons à recréer du lien social. Mais, aussi, ces pratiques permettent parfois la reconnaissance des besoins par des décideurs. C'est un contact direct qui est renoué, sans médiateur (si ce n'est le groupe), naturel dans une démocratie. Si l'objectif professionnel du travailleur social doit être défini, son rôle professionnel de guide du groupe dans le rapprochement avec des élus ne s'improvise pas et il doit non seulement être clarifié auprès des membres du groupe, mais aussi auprès des services et des élus. Notre rôle n'est pas de développer ces contacts a priori, mais d'accompagner le groupe dans son travail propre et de l'aider à mettre en œuvre ses décisions. Ce n'est pas non plus de lui imposer notre propre démarche, car le discours des usagers sera d'autant plus entendu qu'il sera construit et exprimé par eux-mêmes.

\section{CONCLUSION}

Cette intervention aura-t-elle répondu à la question : quelles sont les alliances possibles entre les décideurs, les acteurs et les usagers de l'action sociale? Certainement pas dans toutes ses dimensions, car c'est un sujet vaste qui évolue constamment. Mon objectif était cependant de mettre en évidence l'importance du contexte institutionnel qui existe quel que soit le lieu d'exercice, de même que les enjeux complexes et sous-jacents qui déterminent une succession de décisions politiques dans un domaine de l'action sociale. Le débat pourrait s'engager sur notre place, à nous travailleurs sociaux, dans le processus décisionnel. Nous transmettons des rapports qui 
apportent des informations aux services et aux décideurs, susceptibles d'orienter les décisions. Non seulement nous devons connaître comment ce que nous produisons est utilisé, mais cela ne doit pas nous dispenser de contacts directs avec les décideurs. Indépendamment de cela, je pense fermement que nous avons un rôle particulier à jouer dans le développement de la place des usagers dans le processus de construction d'une politique sociale locale. Nos compétences spécifiques, notamment en travail de groupe, et notre proximité avec les usagers sont des moyens pour élaborer des pratiques qui permettent à des personnes de retrouver leur place de citoyens. Leurs discours propres valent bien nombre de nos rapports!

Catherine MANSON

Assistante sociale Formatrice en travail social École pratique de service social de Paris et Institut de formation sociale des Yvelines

Pontoise, France

\section{Références bibliographiques}

GonTCHAROFF, G. (1988). Guide du partenariat des associations et des pouvoirs publics locaux, Paris, L'Harmattan.

GONTCHAROFF, G. (1985). La décentralisation : l'action sanitaire et sociale, les difficultés d'adaptation, Tome 5, Paris, Syros.

MULLER, P. (1994). Les politiques publiques, Paris, PUF. (Coll. Que sais-je?)

OHNET, J.M. (1996). Histoire de la décentralisation française, Paris, Livre de poche.

RODIER, F. (1987). « Décentralisation de l'action sanitaire et sociale dans le Puy-de-Dôme », Traces, nº 4. 